\title{
Unusual presentation of a cutaneous leiomyoma of the neck simulating a goiter
}

\author{
Kouame Kanga, Komenan Kassi", Kouame Kouassi, Ildevert Patrice Gbery, Jean-Marie \\ Kanga
}

Department of Dermatology and Infectiology, University of Félix Houphouët Boigny-Abidjan, Ivory Coast

\begin{abstract}
We report an unusual case of a large cutaneous leiomyoma. Dermal leiomyomas are rare and benign skin tumors derived from the smooth erector muscles of the hair. The patient was a 12 -year-old child who came in for a consultation on a big cervical tumor of the anterior part of the neck which simulated goiter. The lesion evolved since the patient was five years old; it was consistently firm and was associated with skin ulceration and significant deep suppurations. Computed tomography (CT) scan revealed that this tumor was limited to the soft and cutaneous tissues without invading the thoracic muscles. Surgical treatment allowed the excision of the gelatinous and well vascularized skin tumor measuring about $15 \mathrm{~cm}$ in diameter. Final histological examination confirmed the diagnosis of dermal leiomyoma.
\end{abstract}

Keywords: Leiomyoma; dermis; goiter; surgery

Citation: Kanga K, Kassi K, Kouassi K, Gbery IP, Kanga JM. Unusual presentation of a cutaneous leiomyoma of the neck simulating a goiter. J Surg Dermatol 2018; 3(2): 62; http://dx.doi.org/10.18282/jsd.v1.i3.62.

*Correspondence to: Komenan Kassi, Department of Dermatology and Infectiology, University of Félix Houphouët Boigny-Abijan, Ivory Coast; siskakomlo@yahoo.fr

Received: $8^{\text {th }}$ April 2018; Accepted: $12^{\text {th }}$ June 2018; Published Online: $24^{\text {th }}$ June 2018

\section{Introduction}

Dermal leiomyomas are benign skin tumors derived from the smooth erector muscles of the hair. These cutaneous tumors are rare and are mostly presented as multiple small cutaneous nodules located on the lower or upper limbs of the body; occurrences on the neck are rare and exceptional. Adults are more affected than children, with an average age of 45 years old ${ }^{[1,2]}$. We report an unusual case of cutaneous leiomyoma of the neck in a child.

\section{Ethics Statement}

The patient's legal parents gave their informed consent for the publication of this case.

\section{Case report}

The patient was a male child, 12 years of age who came in for a consultation on a huge tumor located on the anterior part of the neck. The disease started when he was five years old, and was originally a small nodule which appeared on the anterior part of the neck in the thyroid gland projection area. The tumor was mistaken for a long time as goiter and the parents used traditional medicine without success. The tumor became larger and was associated with skin ulcerations, giving rise to suppurations. It was then his parents decided to consult a community health care center nearby.

There, surgical excision was suggested but it was not adhered to due to the lack of financial means. Hence, traditional medicine was pursued and the

Copyright $(9) 2018$ Kanga K, et al. This is an Open Access article distributed under the terms of the Creative Commons Attribution-NonCommercial 4.0 International License (http://creativecommons.org/icenses/ y-nc/4.0/), permitting all non-commercial use, distribution, and reproduction in any medium, provided the original work is properly cited. 
tumor became more ulcerated and suppurative. Faced with a worsening case, they decided to consult the dermatology department of the Teaching Hospital of Treichville for adequate treatment.

Clinical examinations noted a large and oval tumor which began on the anterior part of the neck (between the cricoid cartilage and the presternal area). The relatively large tumor measured about $15 \mathrm{~cm}$ in diameter and extended from the chest region to the epigastric area (Figure 1). Three skin ulcerations were observed on the tumor, giving rise to suppurative fluid on palpation. In addition, the tumor contained blood vessels associated with numerous venous insufficiencies. Doppler echography, computed tomography (CT) scan and X-ray were performed for organ diagnosis to identify if the tumor derived from the thyroid gland. $\mathrm{X}$-ray images revealed that the tumor derived from the cutaneous and subcutaneous tissues without invading the chest muscles and thyroid gland (Figure 2). Histopathological examination performed after excising the tumor concluded a dermal leiomyoma (Figure 3). The wound was concealed via total skin graft taken from the internal part of the two arms and healed without any recurrence after two years of follow-up (Figure 4).

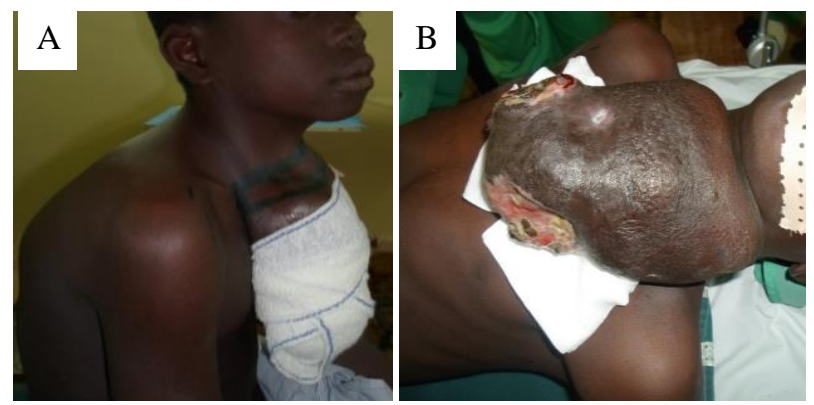

Figure 1. Cutaneous leiomyoma of the anterior part of the neck in a 12-year-old patient (A) Lateral view before treatment, (B) During pre-operation

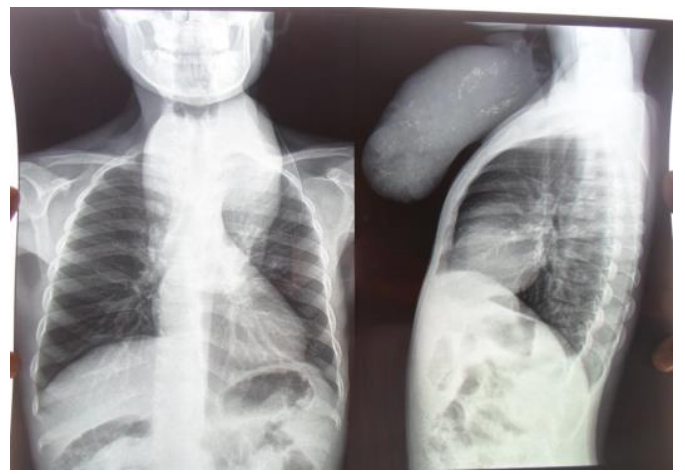

Figure 2. X-ray of the chest showing a cutaneous leiomyoma of the anterior part of the neck (frontal and lateral views)

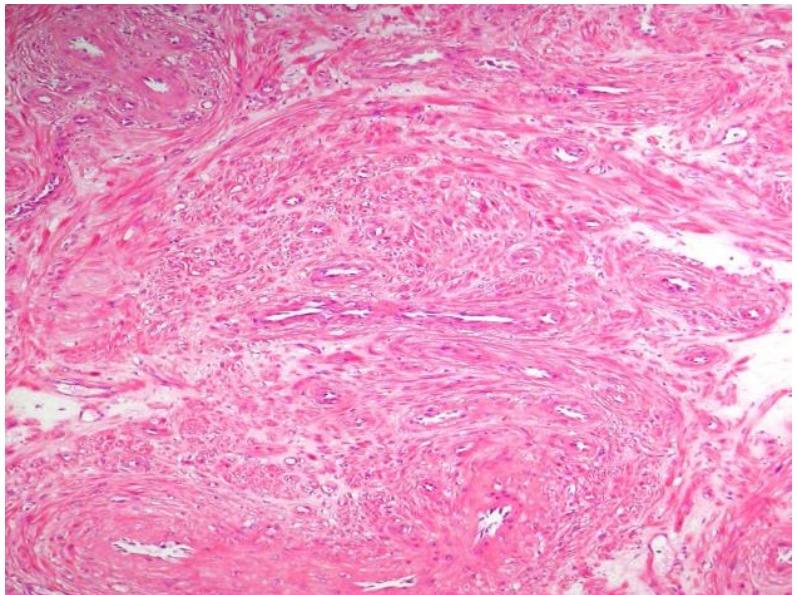

Figure 3. Dermal leiomyoma with vascularized components, no mitotic and atypical cells (HematoxylinEosine staining, magnification $\mathrm{X} 40$ )

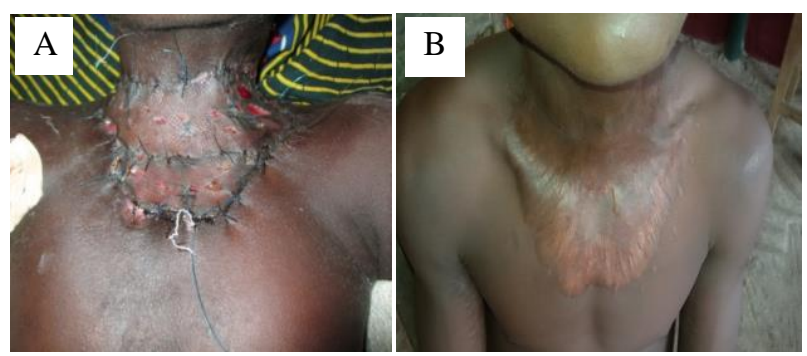

Figure 4. Cutaneous leiomyoma of the anterior part of the neck: (A) immediately after thick skin graft, (B) after complete wound healing

\section{Discussion}

Dermal leiomyomas are uncommon benign tumors derived from erector pili muscles. This tumor is rarely reported in our setting. However, multiple clinical cases were reported in literature. Ghanadan et al. reported a series of 25 cases, which allowed the adoption of a new classification ${ }^{[2]}$.

In our case, the disease was diagnosed in a male child and it started when he was five years old. Male adult patients are more likely affected by the disease, with a mean age of 45 years old ${ }^{[1,2]}$. Cutaneous leiomyoma is exceptional in a child. It usually presents itself as numerous small skin lesions can be painful and are located on the upper or lower limbs (leg or arm) in $52 \%$ of the cases. Occurrences on the neck are rare and exceptional. In literature, previous angioleiomyoma cases have been reported at unusual sites, for instance the lip, oral cavity, and structures such as the epididymis, spermatic cord, and blood vessels ${ }^{[3]}$. In our case, the neck was the manifestation 
site and this could have been derived from blood vessels due to the hyper vascularization of this area of the human body. This type of tumor is mostly painless and extends slowly during several years (ranging from 2 months to 20 years) ${ }^{[1]}$. In our case, it evolved for 7 years (from 5 years old to 12 years old) to reach $15 \mathrm{~cm}$ in diameter associated with the cervical vertebrae - simulating goiter. In fact, this location may explain the delay of diagnosis and the therapeutic behavior observed in the parents. Physical examination of the tumor did not find the tumor moving during swallowing. However, we could detect pulsation on the tumor's lateral basis, which allowed us to deduce an important revascularization of the tumor.

Paraclinical examinations, CT scan, and X-ray confirmed the cutaneous location of the tumor without thyroid gland tumor. Skin biopsy performed for histopathological examination confirmed dermal leiomyoma with vascularized components and without the presence of mitotic cells. Ghanadan et al. have classified leiomyoma into three classes: 1) pillar leioyoma (PLM) which derives from the erector muscles of hair, 2) genital leiomyoma (GLM), and 3) angioeiomyoma (ALM) which derives from smooth muscles of the blood vessels ${ }^{[2]}$. Our case was classified as ALM as the tumor was very well vascularized. This could explain the exceptionally large tumor observed in the seven years of evolution. In spite of this large tumor, we did not find any atypical or mitotic cells. Therefore, it was not a leiomyosarcoma ${ }^{[4]}$.

In terms of treatment, a surgical excision of the tumor was performed under general anesthesia. After one month of wound dressing, good tissue granulation was obtained and the wound was enclosed via a skin graft taken on the internal area of the two arms. The treatment procedure used in our case was in accordance with those found in literature, wherein a large surgical excision is recommended to avoid recurrences ${ }^{[5,6]}$. The specificity of our case was the absence of pain, the location of the tumor, and the tumor size which needed skin graft (Figure 4). In terms of follow-up, we did not find any recurrence after three years and aesthetical functional result was perfect.

\section{Conclusion}

This case of cutaneous leiomyoma is exceptional not only in terms of size and localization, but also in surgical treatment which gave hope of life to our young patient who returned to school after the wound healed.

\section{Acknowledgements}

The author acknowledges all dermatology health practitioners who took care of the patient.

\section{Conflict of interest}

The author declares no potential conflict of interest with respect to the research, authorship, and/or publication of this article.

\section{References}

1. Bhaskar S, Jaiswal AK, Madhu SM, Santosh KV. Unusual presentation of cutaneous leiomyoma. Indian J Dermatol 2014; 59(6): 634.doi: 10.4103/0019-154.143590.

2. Ghanadan A, Abbasi A, Kamyab Hesari K. Cutaneous leiomyoma: Novel histologic findings for classification and diagnosis. Acta Med Iran 2013; 51(1): 19-24.

3. Anandraj VK, Kotasthane D. Cutaneous leiomyoma: A case study with review of literature on rare variants and clinical behavior. Int J Pharm Bio Sci 2015; 6(3): B907B913.

4. Ciurea ME, Georgescu CV, Radu CC, Georgescu CC, Stoica LE. Cutaneous leiomyosarcoma-Case report. J Med Life 2014; 7(2): 270-273.

5. El Morabite K, Hassam B. Nodule sous cutané révélant un angioléiomyome (French) [Subcutaneous nodule revealing an angioleimyoma]. Pan Afr Med J 2013; 15: 48. doi: 10.11604/pamj. 2013.15.48.2823.

6. Hissane EM, Fasla H, Mahdaoui S, Noun M, Hermas S, et al. Léiomyome de la vulve: Á propos d'un cas et revue de la literature (French) [Leiomyoma of the vulva: A case report]. Imagerie de la Femme 2001;21(4):179-181.doi: 10.1016/j.femme.2011.10.009. 